\title{
COMPOSIÇÃO QUÍMICA E ATIVIDADE ANTIOXIDANTE DA ESPÉCIE Lippia thymoides MART. \& SCHAUER CULTIVADA
}

\author{
$\underline{\text { Maíra Meira de Freitas }}^{1}$; Angélica Maria Lucchese ${ }^{2}$ e Horácio Freitas Bomfim ${ }^{3}$
}

1. Bolsista PIBIC/CNPq, Graduanda em Farmácia, Universidade Estadual de Feira de

Santana, e-mail: mairafreitas s2@hotmail.com

2. Orientadora, Departamento de Exatas, Universidade Estadual de Feira de Santana, email: angelica.lucchese@gmail.com

3. Membro do Laboratório de Química de Produtos Naturais e Bioativos, Departamento de Exatas, Universidade Estadual de Feira de Santana, e-mail: hfreitasb@gmail.com

PALAVRAS-CHAVE: fitoquímica, extrato, antioxidante.

\section{INTRODUÇÃO}

A utilização de plantas medicinais e da fitoterapia encontra-se em expansão no Brasil e no mundo, constituindo um mercado farmacêutico altamente promissor (BREVOORT, 1988), tendo em vista a utilização das substâncias como protótipos para o desenvolvimento de fármacos, ou ainda, de medicamentos elaborados exclusivamente à base de extratos vegetais, os fitoterápicos (VIEGAS et al., 2006). Com os avanços tecnológicos e aumento do interesse dos pesquisadores a esse tema, tem crescido a recomendação do uso de plantas medicinais pelos profissionais da saúde com fins curativos(ARNOUS et al., 2005).

Dentre as diversas famílias de interesse medicinal está a família Verbenaceae, com inúmeros relatos de utilização na medicina tradicional. Esta família, incluída na ordem Lamiales, compreende em torno de 36 gêneros e 1000 espécies de distribuição pantropical, mas principalmente neotropical. No Brasil, existem 17 gêneros e aproximadamente 250 espécies, dentre os quais se situa o gênero Lippia. Este gênero reúne cerca de 200 táxons, entre ervas, arbustos e pequenas árvores, distribuídos nos trópicos e subtrópicos das Américas e África, sendo comuns nos cerrados e campos rupestres brasileiros. As espécies deste gênero são ervas ou arbustos e muitas são aromáticas (SOUZA\& LORENZI, 2005), com várias espécies ainda desconhecidas do ponto de vista químico e/ou farmacológico, a exemplo da espécie Lippia insignis, um arbusto aromático nativo do Estado da Bahia, com distribuição na caatinga e cerrado (SALIMENA, MULGURA, 2014).

Plantas do gênero Lippia são comumente usadas pela população para a cura de diversas enfermidades, destacam-se pelo aroma forte e agradável e seu aspecto atrativo no período de floração. Estudos comprovaram que algumas espécies desse gênero possuem principalmente ação como antisséptico e antimicrobiano (COSTA et al., 2002)

Estudos da composição química de espécies de Lippia indicam que este gênero é rico em compostos fenólicos e flavonoides, possuindo assim ação antioxidante (AGUIAR e COSTA, 2005). A oxidação é um processo essencial aos organismos aeróbios e ao nosso metabolismo, sendo os radicais livres produzidos naturalmente, como consequência desse processo de oxidação, ou por alguma disfunção biológica. No organismo, os radicais livres desempenham diversos papéis. Entretanto, o excesso deles pode ser responsável por uma série de efeitos deletérios, como, por exemplo, neoplasias, envelhecimento precoce e problemas cardiovasculares, que podem ser evitadas com a ingestão de substâncias com ação antioxidante (BARREIROS et al., 2006). Isso ocorrerá porque os antioxidantes têm como propriedade doar um elétron para as moléculas já oxidadas e ao se oxidarem permanecem estáveis, ou seja, não se tornam novos radicais livres interrompendo a reação em cadeia (FERREIRA et al., 1997). 
Assim, um dos fatores pela crescente demanda em estudos químicos e de ação biológica com plantas e micro-organismos é a busca por novos fármacos que tenha uma ação antioxidante. Essa área de pesquisa tem um futuro promissor principalmente no território brasileiro que possui uma rica biodiversidade, com variadas espécies endêmicas distribuídas em distintos biomas (NEWMAN et al., 2007).

O cultivo de plantas medicinais envolve a possibilidade de domesticação da espécie a ser utilizada e tal possibilidade implica no domínio tecnológico de todas as etapas de desenvolvimento da espécie. Nem sempre as condições ideais para o desenvolvimento e produção de biomassa são as mais adequadas à produção dos princípios ativos de interesse. Mas, geralmente, existe uma boa adaptação às situações que se assemelham àquela de seu local de origem (REIS; MARIOT, 2015). Desta forma, o objetivo deste trabalho foi avaliar a composição química e a atividade antioxidante da fração em acetato de etila das folhas de Lippia thymoides cultivada.

\section{MATERIAIS E MÉTODOS}

\section{FRACIONAMENTO DO EXTRATO BRUTO}

O extrato metanólico das folhas de Lippia thymoides foi fracionado por partição líquido-líquido. O extrato bruto (331g) foi ressuspenso em metanol:água (8:2 v/v) e extraído com hexano; diclorometano e acetato de etila para obtenção das frações, visando uma semipurificação das substâncias através de suas polaridades. As frações supracitadas depois de obtidas foram concentradas em evaporador rotatório e colocadas para secar em capela de exaustão, enquanto a fração hidrometanólica teve o metanol retirado no evaporador rotatório e a solução final foi liofilizada (Liofilizador Terrone Enterprise I). A fração em acetato de etila (FAC) foi a selecionada para os ensaios posteriores.

\section{DETERMINAÇÃO DO CONTEUDO DE FLAVONOIDES TOTAIS}

$\mathrm{O}$ ter de flavonoides foi também determinado através da espectrofotometria no UV-Vis $(\lambda=425 \mathrm{~nm}$ ), pelo método de Banov (2006), utilizando como padrão quercetina. Os valores obtidos foram calculados por regressão $\left(\mathrm{R}^{2}=0,9978\right)$ através da curva de calibração exposta na figura 2, onde Y é o valor da absorbância e X o valor da concentração em $\mu \mathrm{gEQ} / \mathrm{mL}$ :

\section{DETERMINAÇÃO DA ATIVIDADE ANTIOXIDANTE}

A atividade antioxidante da fração de acetato de etila primeiramente foi determinada pelo método do sequestro de radical livre 1,1-difenil-2-picrilhidrazila (DPPH') segundo Sousa (2007) com algumas adaptações. O valor da absorbância do DPPH foi ajustado para 1,005 em um comprimento de onda de $517 \mathrm{~nm}$, e com uma concentração de $40 \mu \mathrm{g} / \mathrm{mL}$ da solução preparada. Amostras da fração foi preparada nas concentrações de $10 \mu \mathrm{g} / \mathrm{mL} ; 15 \mu \mathrm{g} / \mathrm{mL} ; 20$ $\mu \mathrm{g} / \mathrm{mL} ; 25 \mu \mathrm{g} / \mathrm{mL} ; 30 \mu \mathrm{g} / \mathrm{mL} ; 35 \mu \mathrm{g} / \mathrm{mL}$. O percentual de inibição das amostras foi calculado pela formula que segue: \% Inibição $=[$ Abs DPPH $-($ Abs da Amostra - Abs Controle da Amostra) x 100]/ Abs DPPH

$\mathrm{O}$ valor de $\mathrm{CE}_{50}$ (concentração de antioxidante necessária para seqüestrar $50 \%$ dos radicais livres de DPPH) foi calculado a partir de regressão linear $\left(R^{2}=0,9902\right)$ pela formula que segue, $(\mathrm{Y}=0,5907 \mathrm{X}-7,6892)$. Ácido ascórbico e Trolox foram usados como padrões.

Seguido do teste de DPPH• para avaliação da atividade antioxidante foi feito o teste de inibição de oxidação do $\beta$-caroteno/ác. linoleico, segundo Lima (2008) com algumas 
adaptações. $\mathrm{O}$ valor da absorbância da solução sistema do $\beta$-caroteno/ác. linoleico foi ajustada para 0,6 a $0,7 \mathrm{~nm}$ com um comprimento de onda de $470 \mathrm{~nm}$. As analises foram feitas utilizando as concentrações de extratos de $100 \mu \mathrm{g} / \mathrm{mL} ; 200 \mu \mathrm{g} / \mathrm{mL} ; 300 \mu \mathrm{g} / \mathrm{mL} ; 400 \mu \mathrm{g} / \mathrm{mL}$ e $500 \mu \mathrm{g} / \mathrm{mL}$. Uma solução de trolox com concentração de $0,2 \mathrm{mg} / \mathrm{mL}$ foi empregada como padrão.

\section{RESULTADOS E DISCUSSÃO}

Os dados de teor de extrativos (rendimento), teor de fenólicos e flavonoides da fração em acetato de etila estão apresentados na tabela 1.

Tabela 1. Rendimento (RE), fenólicos e flavonoides totais da fração em acetato de etila

\begin{tabular}{c|c|c|c}
\hline Amostra & $\begin{array}{c}\text { Média } \pm \text { DP } \\
\text { mg EAG/g }\end{array}$ & $\begin{array}{c}\text { Média } \pm \text { DP } \\
\text { mg QUE/g }\end{array}$ & RE \\
\hline FAC & $279,65 \pm 10,72$ & $26,93 \pm 0,81)$ & $29,56 \%$ \\
\hline
\end{tabular}

FAC (Fração em acetato de etila) Fonte: autor, 2017

Os compostos fenólicos, além de proporcionar atividade antioxidante que prolongam o armazenamento de produtos, têm demonstrado importante atividade "in vivo" e podem ser benéficos no controle de diversas doenças relacionadas à formação excessiva de radicais livres que excedem a capacidade de defesa antioxidante do corpo humano como aterosclerose, cataratas e câncer (JARDINI et. al., 2007). O teor de compostos fenólicos e flavonoides totais observados sugerem que esta fração pode ser fonte de compostos antioxidantes. Os dados de atividade antioxidante, avaliada pelo seqüestro do radical livre DPPH, da fração das folhas e dos controles positivos (ácido ascórbico e Trolox) estão apresentados na Tabela 2.

Tabela 2. Valores médios de $\mathrm{CE}_{50}$ da fração em acetato de etila, do ácido ascórbico e Trolox em $\mu \mathrm{g} / \mathrm{mL}$

\begin{tabular}{c|c|c|c}
\hline \multirow{2}{*}{ Amostras } & $\begin{array}{c}\text { FAC } \\
\text { Média } \pm \text { DP }\end{array}$ & $\begin{array}{c}\text { Trolox } \\
\text { Média } \pm \text { DP }\end{array}$ & $\begin{array}{c}\text { Ácido } \\
\text { Ascórbico } \\
\text { Média } \pm \text { DP }\end{array}$ \\
\hline$* \mathbf{C E}_{\mathbf{5 0}}$ & $18,03 \pm 1,47$ & $9,49 \pm 0,67$ & $7,12 \pm 0,66$ \\
\hline
\end{tabular}

pelo sequestro de radical livre. FAC (Fração em acetato de etila) Fonte: autor, 2017

Quanto menor é a $\mathrm{CE}_{50}$ de uma amostra, maior é a sua atividade antioxidante, já que a mesma necessita de concentrações menores para um maior consumo de DPPH'. Verifica-se que a fração em acetato de etila de folhas de Lippia thymoides é capaz de sequestrar o radical livre $\mathrm{DPPH}^{\circ}$, entretanto com valores inferiores aos antioxidantes sintéticos, ácido ascórbico e o trolox.

A capacidade desta fração em inibir a oxidação no sistema $\beta$-caroteno/ác. linoleico, está expressa na tabela 2, bem como do controle positivo trolox. 
Tabela 2 - Porcentagem inibição de oxidação [proteção] para a fração em acetato de etila no sistema $\beta$-caroteno/ácido linoleico.

\begin{tabular}{c|c|c|c|c|c|c}
\hline Amostras & $\begin{array}{c}100 \\
\mu \mathrm{g} / \mathrm{mL}\end{array}$ & $\begin{array}{c}200 \\
\mu \mathrm{g} / \mathrm{mL}\end{array}$ & $\begin{array}{c}300 \\
\mu \mathrm{g} / \mathrm{mL}\end{array}$ & $\begin{array}{c}400 \\
\mu \mathrm{g} / \mathrm{mL}\end{array}$ & $\begin{array}{c}500 \\
\mu \mathrm{g} / \mathrm{mL}\end{array}$ & $\begin{array}{c}\text { Trolox } \\
200 \\
\mu \mathrm{g} / \mathrm{mL}\end{array}$ \\
\hline FAC & $6,17 \%$ & $19,40 \%$ & $29,90 \%$ & $36,11 \%$ & $27,77 \%$ & $83,64 \%$ \\
\hline
\end{tabular}

FAC (Fração em acetato de etila) Trolox (padrão utilizado nas análises). Fonte: autor, 2017

A partir da curva cinética obtida é possível calcular os fatores cinéticos do extrato (YANISHILIEVA \& MARINOVA, 1995). O fator F1 (calculando no período entre 15 e 45 minutos de reação) mensura a eficiência do antioxidante em bloquear a reação em cadeia, por meio da inativação dos radicais peróxidos, seriam os chamados antioxidantes primários. Enquanto o fator F2 (calculado entre 75 e $105 \mathrm{~min}$.) está associado a capacidade do antioxidante de participar de outras reações como, por exemplo, a decomposição dos produtos secundários da oxidação, como os radicais hidroperóxidos. Quanto menor o valor de F1, mais eficientes são os compostos da amostra em retardar ou inibir o processo oxidativo. No entanto, nos dois casos, se o valor da razão entre as tangentes das curvas do extrato e do controle forem menores que 1, atuam efetivamente como antioxidantes; se os valores forem maiores que 1, então não há um resultado satisfatório quanto a atividade antioxidante nesse intervalo, e o extrato pode atuar como pró-oxidante.

Tabela 4- Parâmetros cinéticos do potencial antioxidante no sistema $\beta$-caroteno/ácido linoléico, para a fração em acetato de etila das folhas de Lippia thymoides

\begin{tabular}{|c|c|c|c|c|c|c|c|}
\hline \multirow{3}{*}{ Órgãos } & \multicolumn{7}{|c|}{ Concentração $\mu \mathrm{g} / \mathrm{mL}$} \\
\hline & \multicolumn{2}{|r|}{100} & \multicolumn{2}{|c|}{200} & 300 & 400 & 500 \\
\hline & & $\mathrm{F} 2$ & & $\mathrm{~F} 2$ & $\mathrm{~F} 1 \quad \mathrm{~F} 2$ & $\mathrm{~F} 1 \quad \mathrm{~F} 2$ & $\mathrm{~F} 1 \quad \mathrm{~F} 2$ \\
\hline FAC & 0,2 & 2,9 & 0,4 & 2,6 & $0,4 \quad 2,2$ & $0,3 \quad 2,1$ & $0,4 \quad 2,1$ \\
\hline Trolox & & NR & 0,1 & 0,2 & NR & NR & NR \\
\hline
\end{tabular}

FAC (Fração em acetato de etila) F1 = fator cinético 1 (tempo compreendido entre 15 a 45 minutos) e F2 = fator cinético 2 (tempo compreendido entre 75 a 105 minutos). Trolox (padrão) NR - Não realizada. Fonte: autor, 2017.

Todas as concentrações da fração em acetato de etila de Lippia thymoides apresentaram os valores do fator F1 menores que 1, ou seja, há capacidade de degradar os radicais peroxido. Entretanto, apresentaram valores de F2 maiores que 1, revelando que os compostos antioxidantes presente participam de outras reações, formando espécies radicalares que aceleram o processo oxidativo do sistema, consequentemente não sendo efetivos na inativação de produtos secundários da reação, como hidroperóxidos, exibindo, portanto, uma atividade pró-oxidante na etapa de propagação da cadeia oxidativa.

Dados anteriormente obtidos em nosso grupo de pesquisa com esta fração, mas da espécie coletada em habitat natural, indicaram seu potencial antioxidante, apresentando valor de $\mathrm{CE}_{50}$ de 5,7 $\pm 0,4 \mu \mathrm{g} / \mathrm{mL}$ no sequestro do radical DPPH e 37,9\% de inibição da oxidação no sistema $\beta$-caroteno/ác. linoleico para uma concentração de $1000 \mu \mathrm{g} / \mathrm{mL}$ (SOUZA et al., 2015). O cultivo de plantas medicinais envolve a possibilidade de domesticação da espécie a ser utilizada e tal possibilidade implica no domínio tecnológico de todas as etapas de desenvolvimento da espécie. Nem sempre as condições ideais para o desenvolvimento e produção de biomassa são as mais adequadas à produção dos princípios ativos de interesse, embora geralmente exista uma boa adaptação às situações que se assemelham àquela de seu 
local de origem (REIS; MARIOT, 2015). Por comparação com os dados supracitados podemos sugerir que espécie cultivada manteve seu potencial antioxidante em valores próximos aos anteriormente obtidos, se mostrando promissora na busca de novos agentes antioxidantes.

\section{CONSIDERAÇÕES FINAIS}

Os teores de compostos fenólicos e flavonoides, bem como os dados de atividade antioxidante, sugerem que a espécie cultivada possui potencial na busca de novas substâncias com atividade antioxidante. A purificação desta fração, que se mostrou promissora, deve ser conduzida visando o isolamento dos metabólitos com atividade antioxidante.

\section{REFERÊNCIAS}

AGUIAR, J. S.; COSTA, M. C. C. D. Lippia alba (Mill) N.E. Brown (Verbenaceae): levantamento de publicações nas áreas química, agronômica e farmacológica, no período de 1979 a 2004. Revista Brasileira de Plantas Medicinais. v. 8, n. 1, p. 79-84, 2005.

ARNOUS, A.H; Santos A.S, BEINNER, R.P.C. Plantas Medicinais De Uso Caseiro: Conhecimento Popular e Interesse Por Cultivo Comunitário. Revista Espaço Para a Saúde. v.6, n.2, p 1-6, jun.2005.

BANOV, D. et al. Caracterização do extrato seco de Ginkgo biloba L. em formulação de uso tópico. Acta Form. Bonaerense 25(2) : 219-24

Barreiros ALBS, David JM, David JP. Estresse oxidativo: relação entre geração de espécies reativas e defesa do organismo. Quim Nova. 2006;29(1):113-23.

BOSCOLO, O. H. et al. Potencial antioxidante de algumas plantas de restingacitadas como medicinais. Revista Brasileira de Plantas Medicinais. v. 9, p. 8-12, 2007.

BREVOORT, P. The Booming U.S. Botanical market.A new overview.Herbalgram, n. 44, p. 33-46, 1988.

COSTA, S. M. O.; LEMOS, T. L. G.; Pessoa, O. D. L.; Assunção, J. C. C.; Braz-Filho, R.;Rev. Bras. Farmacogn. 2002,12, 66.

MERCÊS, Manuela D. Potencial antimicrobiano e atividade antioxidante de espécies do gênero lippia (verbenaceae) do semi-árido baiano. Dissertação-Mestrado em Biotecnologia, Universidade Estadual de Feira de Santana, 2011.

EINSENBERG, D.M.; DAVIS, R.B.; ETTNWR, S.; APPEL, S.; WILEY, S.; ROMPAY, M.V.; KESSLER, R.C. Trends in alternative medicine use in the United States, 19901997.Journalof American Medical Association, v. 280, n. 18, p. 1569-75, 1998.

FERREIRA, A.L.A.; MATSUBARA, L.S.. Radicais livres: conceitos, doenças relacionadas, sistema de defesa e estresse oxidativo. Rev. Assoc. Med. Bras., São Paulo,v. 43, n. 1, p. 6168, 1997.

NEWMAN, D.J.; CRAGG, G.M. Natural products as sources of new drugs over the last 25 years.Journalof Natural Products. v. 70, p. 461-477, 2007.

PERES, M. T. L. P et al. Estudos químicos e biológicos de micrograma (Larsal Fisch.) Copel (Polypodiaceal). Química Nova, v. 32, n.4, p. 897-901.

REIS , M. S. ; MARIOT, A. Diversidade natural e aspectos agronômicos de plantas medicinais. In: SIMÕES, C. M. O.; SCHENKEL, E. P.; GOSMANN, G. et al. (Org.). Farmacognosia: da planta ao medicamento. Porto alegre: UFRGS: UFSC, 1999. p. 39 -60.

SALIMENA, F.R.G.; Mulgura, M. Lippia in Lista de Espécies da Flora do Brasil. Jardim Botânico do Rio de Janeiro. Disponível em: <http://floradobrasil.jbrj.gov.br/jabot/floradobrasil/FB21444>. Acesso em: 16 Ago. 2014 
SOUZA, V.C.; LORENZI, H. Botânica Sistemática. Nova Odessa: Instituto Plantarum de Estudos da Flora Ltda, 2005.

SOUSA, C. M. M. et al. Fenóis totais e atividade antioxidante de cinco plantas medicinais. Química Nova, v. 30, n. 2, p. 351-355, 2007.

VIEGAS JUNIOR, C.; BOLZANI, V. S; BARREIRO, E. J. Os produtos naturais e a química medicinal moderna Química Nova. v. 29, n. 2, p. 326-337, 2006.

SOUZA, S. F. et al. Pharmacological Basis for Traditional Use of the Lippia thymoides.

Hindawi. 2015. Disponivel em: <http://dx.doi.org/10.1155/2015/463248>. Acesso em 28 Jul. 2017. 